\title{
Formation of Liesegang patterns
}

\author{
Zoltán Rácz \\ Institute for Theoretical Physics, Eötvös University \\ Pázmány sétány 1/a, 1117 Budapest, Hungary
}

\begin{abstract}
It has been recently shown that precipitation bands characteristic of Liesegang patterns emerge from spinodal decomposition of reaction products in the wake of moving reaction fronts. This mechanism explains the geometric sequence of band positions $x_{n} \sim Q(1+p)^{n}$ and, furthermore, it yields a spacing coefficient $p$ that is in agreement with the experimentally observed MatalonPackter law. Here I examine the assumptions underlying this theory and discuss the choice of input parameters that leads to experimentally observable patterns. I also show that the so called width law relating the position and the width of the bands $w_{n} \sim x_{n}$ follows naturally from this theory.
\end{abstract}

\section{INTRODUCTION}

Formation of precipitation patterns in the wake of moving reaction fronts (known as the Liesegang phenomenon) has been studied for more than a century [1] 3 . The motivation for these studies has been diverse, coming from the importance of related practical problems such as crystal growth in gels, as well as from the fascination with a complex pattern that has eluded a clean-cut explanation (e.g. agate rocks are believed to display Liesegang patterns). From a theoretical point of view, the main factor in the popularity was the belief that much can be learned about the details of precipitation processes (nucleation, growth, coagulation, etc.) by investigating the instabilities underlying this phenomenon. Currently, the Liesegang phenomenon is mainly studied as a nontrivial example of pattern formation in the wake of a moving front [ [- 6] and there are speculations about the possibility of creating complex mesoscopic structures using this rather inexpensive process.

Liesegang patterns are easy to produce (Fig.1 shows a particular experiment that we shall have in mind in the following discussion). The main ingredients are two chemicals $A$ and $B$ yielding a reaction product $A+B \rightarrow$ $C$ that forms a nonsoluble precipitate $C \rightarrow D$ under appropriate conditions $\left[A=\mathrm{NaOH}, \mathrm{B}=\mathrm{MgCl}_{2}\right.$ and $D=M g(O H)_{2}$ in Fig.1]. The reagents are separated initially with one of them ( $B$, inner electrolyte) dissolved in a gel and placed in a test tube. Then at time $t=0$ an aqueous solution of the other reagent $(A$, outer electrolyte) is poured over the gel. The initial concentration $a_{0}$ of $A$ is chosen to be much larger than that of $B$ (typically $a_{0} / b_{0} \approx 10^{2}$ ), thus $A$ diffuses into the gel and a reaction front moves down the tube. Behind the front, a series of stationary precipitation zones (Liesegang bands) appear at positions $x_{n}$ ( $x_{n}$ is measured from the interface between the gel and the aqueous solution; $n=1,2, \ldots, 10-20$, typically). A band appears in a rather short time-interval thus the time of the appearance $t_{n}$ of the $n$-th band is also a well defined, experi- mentally measurable quantity. Finally, the widths of the bands $w_{n}$ can also be determined in order to characterize the pattern in more detail.

JPEG file attached

FIG. 1. Liesegang patterns obtained with reagents $A=N a O H$ and $B=M g C l_{2}$ in polyvinylalcohol gel. The white precipitate is $\mathrm{D}=\mathrm{Mg}(\mathrm{OH})_{2}$. The height of the columns is $30 \mathrm{~cm}$ and it takes about a $1-2$ weeks for the patterns to form. The columns show different patterns due to the difference in the initial concentrations of the outer electrolyte $\mathrm{NaOH}$. The experiments were carried out by $\mathrm{M}$. Zrínyi (Technical University of Budapest).

The experimentally measured quantities $\left(x_{n}, t_{n}\right.$ and $w_{n}$ ) in regular Liesegang patterns satisfy the following time-, spacing-, and width laws.

- Time law [7]:

$$
x_{n} \sim \sqrt{t_{n}} .
$$

This law is satisfied in all the experiments where it was measured and it appears to be a direct consequence of the diffusive dynamics of the reagents.

- Spacing law [8]: The positions of the bands form a geometric series to a good approximation

$$
x_{n} \sim Q(1+p)^{n}
$$

where $p>0$ is the spacing coefficient while $Q$ is the amplitude of the spacing law. The quantitative experimental observations concern mainly this law. More detailed works go past the confirmation of the existence of the geometric series and study the dependence of the spacing coefficient on 
$a_{0}$ and $b_{0}$. The results can be summarized in a relatively simple expression usually referred to as the Matalon-Packter law [9,10]:

$$
p=F\left(b_{0}\right)+G\left(b_{0}\right) \frac{b_{0}}{a_{0}}
$$

where $F$ and $G$ are decreasing functions of their argument $b_{0}$.

- Width law 11]:

$$
w_{n} \sim x_{n}
$$

This is the least established law since there are problems with both the definition and the measurement (fluctuations) of the width. Recent, good quality data [12] does support, however, the validity of 4 ).

It should be clear that (14) summarizes only those properties of Liesegang patterns that are common in a large number of experimental observations. There is a wealth of additional data on various details such as e.g. the secondary structures or the irregular band spacing [2]. These features, however, appear to be peculiarities of given systems. It is hard to characterize them and their reproducibility is often problematic as well. In view of this, it is not surprising that the theoretical explanations of Liesegang phenomena have been mainly concerned with the derivation of (11-4).

The theoretical approaches to quasiperiodic precipitation have a long history and the two main lines of thoughts are called as pre- and post-nucleation theories (for a brief overview see [6]). They all share the assumption that the precipitate appears as the system goes through some nucleation or coagulation thresholds. The differences are in the details of treating the intermediate steps "...C..." in the chain of reactions $A+B \rightarrow \ldots C \ldots \rightarrow$ $D$ producing the precipitate $D$. In general, all the theories can explain the emergence of distinct bands but only the pre-nucleation theories can account «, $4,5,13,15$ for the time- and spacing laws of normal patterns. These theories are rather complicated, however, and have been developed only recently [6] to a level that the dependence of $p$ on the initial concentrations $a_{0}$ and $b_{0}$ can be investigated quantitatively, and connection can be made to the Matalon-Packter law [9,10].

Unfortunately, there are several problems with the theories mentioned above. First, they employ a large number of parameters and some of these parameters are hard to grasp theoretically and impossible to control experimentally (an example is the lower threshold in the density of $C$-s below which aggregation $C+D \rightarrow 2 D$ ceases [5]). Second, some of the mechanisms invoked in the explanations are too detailed and tailored to a given system in contrast to the generality of the resulting pattern in diverse systems. A real drawback of the too detailed description is that quantitative deductions are difficult to make even with the present computer power [4. A final problem we should mention is the absence of an unambiguous derivation of the width law in any of the theories.

In order to avoid the above problems, we have recently developed a simple model of band formation [16] based on the assumption that the main ingredients of a macroscopic description should be the presence of a moving reaction front and the phase separation that takes place behind the front. This theory contains a minimal number of parameters, it accounts for the spacing law, and it is simple enough that the existence of the Matalon-Packter law can be established numerically. The apparent success warrants a closer look at the model and, in this lecture, I will describe in detail how one arrives at such a model and what are the underlying assumptions of the theory. Then I would like to discuss the choice of input parameters that yield experimentally observable patterns and, finally, I will show that the derivation of width law is straightforward in this theory.

\section{THE MODEL}

Let us begin building the model by taking a look at Fig.1. It shows alternating high- and low-density regions of the chemical $\mathrm{Mg}(\mathrm{OH})_{2}$ and the systems appear to be a quasi-steady state (actually, there are experiments that suggest that the pattern does not change over a 30 years period [2]). We shall take this picture as an evidence that phase separation [17] underlies the formation of bands and, furthermore, that the phase separation takes place at a very low effective temperature (no coarsening is observed).

The phase separation, of course, must be preceded by the production of $C$-s. This is the least understood part of the process and it is particular to each system. What is clear is that due to the condition $a_{0} \gg b_{0}$ a reaction front $(A+B \rightarrow$ something $)$ moves down the tube diffusively (note that this is the point where the role of the gel is important since it prevents convective motion). The result of the reaction may be rather complex (intermediate products, sol formation, etc.) and one of our main assumptions is that all these are irrelevant details on a macroscopic level. Accordingly, the production of $C$ will be assumed to be describable by the simplest reaction scheme $A+B \rightarrow C$.

Once $A+B \rightarrow C$ is assumed, the properties of the front and the production of $C$-s are known [18]. Namely, the front moves diffusively with its position given by $x_{f}=\sqrt{2 D_{f} t}$, the production of $C$-s is restricted to a slowly widening narrow interval $\left[w_{f}(t)=w_{0} t^{1 / 6}\right]$ around 
$x_{f}$, and the rate of production $S(x, t)$ of $C$-s can be approximated by a gaussian (the actual form is not a gaussian, see 19] for details about a non-moving front)

$$
S(x, t)=\frac{S_{0}}{t^{2 / 3}} \exp \left[-\frac{\left[x-x_{f}(t)\right]^{2}}{2 w_{f}^{2}(t)}\right] .
$$

The parameter $D_{f}$ can be expressed through $a_{0}, b_{0}$, and the diffusion coefficients of the reagents $\left(D_{a}, D_{b}\right)$ while $S_{0}$ and $w_{0}$ depends also on the rate constant, $k$, of the reaction $A+B \rightarrow C$.

An important property of the front is that it leaves behind a constant density $c_{0}$ of $C$-s [6] and $c_{0}$ depends only on $a_{0}, b_{0}, D_{a}$ and $D_{b}$. This is important because the relevant parameters in the phase separation are $D_{f}$ and $c_{0}$ (where and how much of the $C$-s are produced 20]) and thus the least available parameter $(k)$ does not play a significant role in the pattern formation.

Having a description of the production of $C$-s, we must now turn to the dynamics of their phase separation. Since the emerging pattern is macroscopic, we shall assume that, on a coarse-grained level, the phase separation can be described by the simplest 'hydrodynamical' equation that respects the conservation of $C$-s. This is the CahnHilliard equation [21] or, in other context, it is the equation for model B in critical dynamics [22]. This equation, however, requires the knowledge of the free-energy density $(\mathcal{F})$ of the system. For a homogeneous system, $\mathcal{F}$ must have two minima corresponding to the low- $\left(c_{l}\right)$ and high-density $\left(c_{h}\right)$ states being in equilibrium (Fig.1). The simplest form of $\mathcal{F}$ having this property and containing a minimal number of parameters is the Landau-Ginzburg free energy (Fig.2)

$$
\mathcal{F}=-\frac{1}{2} \varepsilon m^{2}+\frac{1}{4} \gamma m^{4}+\frac{1}{2} \sigma(\nabla m)^{2},
$$

where $m=c-\left(c_{l}+c_{h}\right) / 2$ is the density, $c$, of the $C$-s measured from the average of the two steady state values (we are following the notation in [16] where the 'magnetic language' has its origin in a connection to Ising lattice gases). The parameters $\varepsilon, \gamma$, and $\sigma$ are system dependent with $\varepsilon>0$ ensuring that the system is in the phase-separating regime, $\sigma>0$ provides stability against short-wavelength fluctuations, and requiring $\sqrt{\varepsilon / \gamma}=\left(c_{h}-c_{l}\right) / 2$ fixes the minima of $\mathcal{F}$ at $\pm m_{e}$ corresponding to $c_{l}$ and $c_{h}$. Note that the $m \rightarrow-m$ symmetry is usually not present in a real system and $\mathcal{F}$ could contain e.g. an $m^{3}$ term. The presence or absence of the $m \rightarrow-m$ symmetry, however, is not relevant for the discussion that follows.

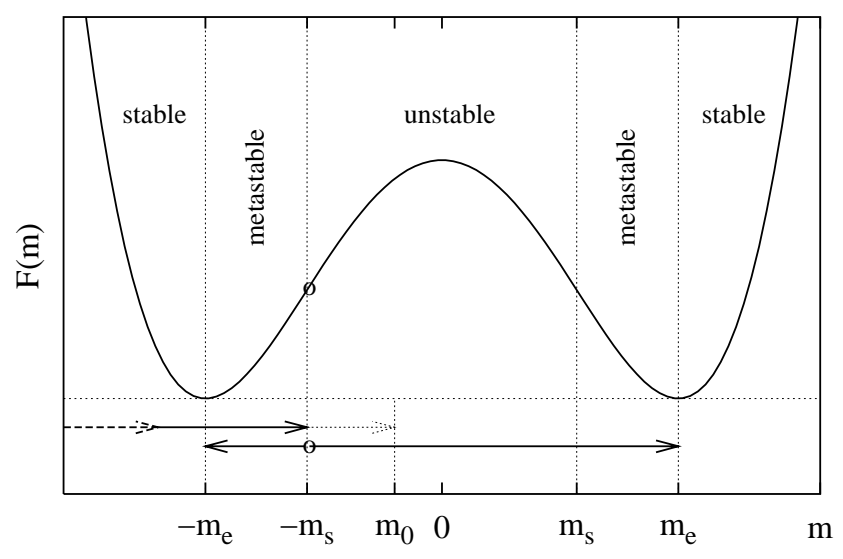

FIG. 2. The homogeneous part of the free energy as a function of $m=c-\left(c_{l}+c_{h}\right) / 2$. The phase separation is an activated process in the metastable regimes while it goes by spinodal decomposition in the (linearly) unstable regime. The spinodal point (o) separates these regimes. The arrows are meant to illustrate how the density in the front increases towards $m_{0}$ and how the phase separation to the steady states $\left( \pm m_{e}\right)$ takes place when the density reaches the spinodal value $-m_{s}$.

Using (6) and including the source term, the CahnHilliard equation takes the form

$$
\partial_{t} m=-\lambda \Delta\left(\epsilon m-\gamma m^{3}+\sigma \Delta m\right)+S .
$$

were $\lambda$ is a kinetic coefficient. The above equation should contain two noise terms. One of them should be the thermal noise while the other should originate in the chemical reaction that creates the source term. Both of these noise terms are omitted here. The reason for neglecting the thermal noise is the low effective temperature of the phase separation as discussed in connection with Fig.1. The noise in $S$, on the other hand, is dropped since the $A+B \rightarrow C$ type reaction fronts have been shown to be mean-field like above dimension two [23].

The absence of noise means that the phase separation can occur only through spinodal decomposition [17,24]. Thus the assumption behind omitting the noises is that the characteristic time of nucleation is much larger than the time needed by the front to increase the density of $C$-s beyond the spinodal value $\left(-m_{s}\right.$ in Fig.2) where the system is unstable against linear perturbations. Since there are examples where the bands appear to be formed by nucleation and growth [2], the spinodal decomposition scenario is clearly not universally applicable, and one should explore the effects of including noise (this becomes, however, an order of magnitude harder problem).

Eq.(7) together with the form of the source (5) defines now our model 16] that produces regular Liesegang patterns (Fig.3) satisfying the spacing law (2) and, furthermore, the spacing coefficient is in agreement with the Matalon-Packter law (3). Fig.3 shows a rather general 
picture that is instructive in understanding the pattern formation. The last band acts as a sink for neighboring particles above $-m_{e}\left(c_{l}\right)$ density. Thus the $C$-s produced in the front end up increasing the width of the last band. This continues until the front moves far enough so that the density in it reaches the spinodal value. Then the spinodal instability sets in and a new band appears. Remarkably, the above picture is rather similar to the phenomenological 'nucleation and growth' scenario [6] with the density at the spinodal point playing the role of threshold density for nucleation. It is thus not entirely surprising that both of these theories do equally well in producing the spacing- and the Matalon-Packter law.

One should note that the actual form of $\mathcal{F}$ does not play an important role in the picture developed above. The crucial feature is the existence of a spinodal density above which phase separation occurs. This is the meaning of our previous remark about the irrelevance of the $m^{3}$ term in the free energy (of course, one should also realize that explanations of details in experiments may require the inclusion of such terms).

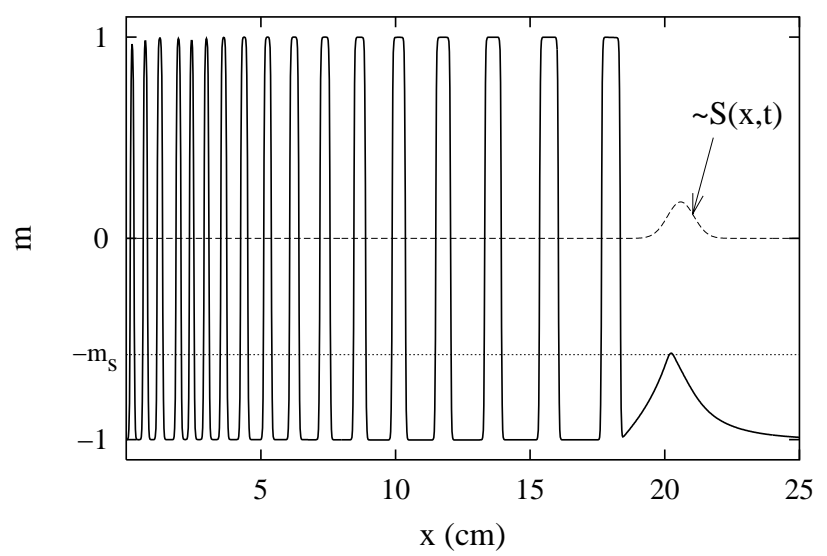

FIG. 3. Liesegang pattern obtained for front parameters $D_{f}=21.72, w_{0}=4.54$, and $S_{0}=0.181$ with length, time, and $m$ (concentration) measured in units of $\sqrt{\sigma / \epsilon}=2 \cdot 10^{-4} \mathrm{~m}$, $\sigma /\left(\lambda \epsilon^{2}\right)=40 \mathrm{~s}$, and $\sqrt{\epsilon / \gamma}$, respectively. The dashed line denotes the rate of production of $C$-s $(S)$, measured in units of $\lambda \epsilon^{5 / 2} /\left(\gamma^{1 / 2} \sigma\right)$ and magnified by a factor $2 \cdot 10^{5}$. The dotted line represents the density at spinodal point, $-m_{s}=-1 / \sqrt{3}$.

\section{CHOICE OF PARAMETERS}

Fig. 3 shows the results of numerical solution of eq.(7) with the same parameter values as in Fig.3 of ref. 16. but stopped at an earlier time so that the visual similarity to the experiments (number of bands in Fig.1) would be greater. In this section, we shall examine whether the parameters used for obtaining this resemblance have any relevance to real Liesegang phenomena.
The experimental patterns have a total length of about $\ell_{\text {exp }} \approx 0.2 \mathrm{~m}$ and the time of producing such a pattern is about 1-2 weeks (we shall take $\tau_{\exp } \approx 10^{6} \mathrm{~s}$ ). Since our model has a length-scale $\ell_{t h}=\sqrt{\sigma / \varepsilon}$ and a time-scale $\tau_{t h}=\sigma /\left(\lambda \varepsilon^{2}\right)$, they can be chosen so $\left[\sqrt{\sigma / \epsilon}=2 \cdot 10^{-4} \mathrm{~m}\right.$ and $\left.\sigma /\left(\lambda \epsilon^{2}\right)=40 \mathrm{~s}\right]$ that $\ell_{\exp } \approx \ell_{t h}$ and $\tau_{\exp } \approx \tau_{t h}$. Once we have chosen $\ell_{t h}$ and $\tau_{t h}$ we can start to calculate other quantities and see if they have reasonable values.

It is clear from Fig. 3 that the widths of the bands are in agreement with the experiments, they are of the order of a few $\mathrm{mm}$ at the beginning and approach to $\sim 1 \mathrm{~cm}$ at the end. The width of the front is also of the order of $1 \mathrm{~cm}$ after $10^{6} \mathrm{~s}$. Unfortunately, there is no information on the reaction zone in this system. In a study of a different system 25] it was found that $w_{f}(\mathrm{t}=2$ hours $) \approx 2 \mathrm{~mm}$. Extrapolating this result to $t=10^{6} \mathrm{~s}$ one finds $w_{f} \approx 1 \mathrm{~cm}$ [note that the exponent of the increase of $w_{f}(t) \sim t^{1 / 6}$ is small] in agreement with the observed value.

Next we calculate the diffusion coefficient of the front, $D_{f}=21.72 \cdot \ell_{t h}^{2} / \tau_{t h} \approx 2 \cdot 10^{-8} \mathrm{~m}^{2} / \mathrm{s}$. This value appears to be an order of magnitude larger than the usual ionic diffusion coefficients $\left(D \approx 10^{-9} \mathrm{~m}^{2} / \mathrm{s}\right)$. One should remember, however, that Fig.3 is the result for initial conditions $a_{0} / b_{0}=10^{2}$ [16] and, for this ratio of $a_{0} / b_{0}$, the diffusion coefficient of the front $D_{f}$ is about 10 times larger than $D_{a}\left(D_{f} / D_{a} \approx 10\right.$ see Fig.4 in [6] $)$. Thus $D_{f}$ also comes out to be the right order of magnitude.

We do not have information on the amplitude $\left(S_{0}\right)$ of the source but, once the concentrations $\left(a_{0}, b_{0}\right)$ are given and $D_{f}$ and $w_{f}$ are known then $S_{0}$ is fixed by the conservation law for the $C$-s. Thus the correct order of magnitude for $D_{f}$ and $w_{f}$ should ensure that $S_{0}$ is also of right order of magnitude.

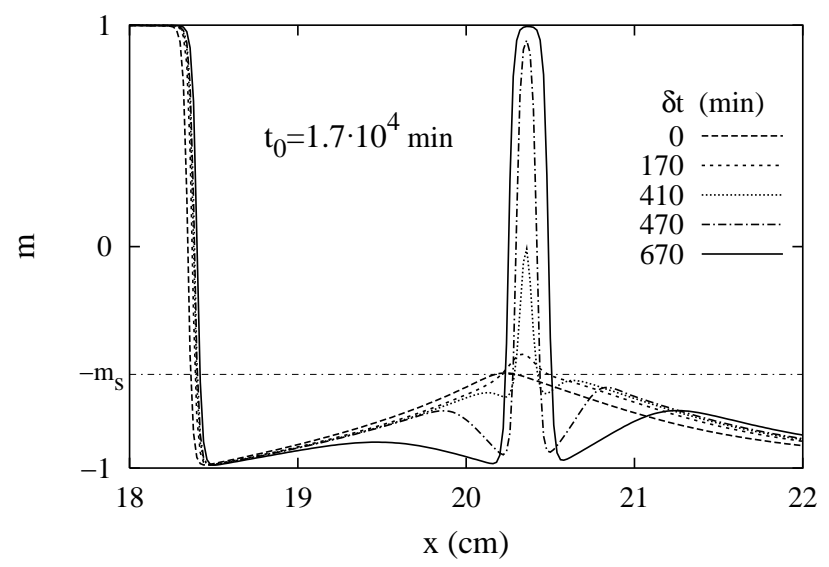

FIG. 4. Details of the time-evolution of the formation of the last band in Fig.3. The time $t_{0}$ is the moment when the concentration at the front reached the spinodal value and $\delta t$ is measured from $t_{0}$.

Finally, we shall calculate the time it takes for a band 
to form. It is well known that the bands appear rather quickly. From the visual notice of the beginning of the band formation it takes about $\tau_{i n i}=30-60$ minutes for the band to be clearly seen and then it takes much longer to increase its width to the final value. In order to calculate $\tau_{i n i}$ let us consider the formation of the last band in Fig.3 (see Fig.4). The lower limit of the density that can be visually noticed is, of course, not well defined. We shall assume that this density corresponds to $m=0$ i.e. it is the halfway density from $c_{l}$ to $c_{h}$. This means that we see the beginnings of the band at $\delta t=410 \mathrm{~min}$ and the density reaches well above $90 \%$ of its final value by $\delta t=470 \mathrm{~min}$. Consequently, we obtain again an estimate $\left(\tau_{\text {ini }} \approx 60 \mathrm{~min}\right)$ for an observed quantity that agrees with the experiments. As a result of the above estimates, we feel that the parameters in our model can indeed be chosen so that they are relevant to real Liesegang experiments.

\section{WIDTH LAW}

The width law is problematic from experimental point of view since the fluctuations in the widths appear to be large. Part of the difficulties are undoubtedly due to the fact that the boundaries of the bands are not sharply defined and high-resolution digitizing methods are needed in a precise analysis. The most thorough experiment to date has been carried out recently [12] with the result $w_{n} \sim x_{n}^{\alpha}$ where $\alpha \approx 0.9-1.0$.

As to the theories, they also have their share of difficulties since, on a microscopic level, the growth of the width involves precipitation processes in the presence of large concentration gradients, while a macroscopic treatment must elaborate on the dynamics of the interfaces between two phases. Accordingly, there are only a few works to report on. Dee [4] used reaction-diffusion equations supplemented by terms coming from nucleation and growth processes and obtained $w_{n} \sim x_{n}$ from a rather limited (6 bands) numerical result. Chopard et al. [5] employed cellular automata simulations of a phenomenological version of the microscopic processes and found $w_{n} \sim x_{n}^{\alpha}$ with $\alpha \approx 0.5-0.6$. Finally, Droz et al. 12] combined scaling considerations with the conservation law for the number of $C$ particles to obtain $\alpha$ in terms of the scaling properties of the density of precipitates in the bands. Assuming constant density they found $\alpha=1$. Our derivation below parallels this last work in that the same conservation law is one of the main ingredient in it.

In our theory, the derivation of the width law is straightforward. One combines the facts that (i) the reaction front leaves behind a constant density $\left(c_{0}\right)$ of $C$-s, (ii) the $C$-s segregate into low $\left(c_{l}\right)$ and high $\left(c_{h}\right)$ density bands, (iii) the number of $C$-s is conserved in the segregation process; and writes down the equation expressing the conservation of $C$-s

$$
\left(x_{n+1}-x_{n}\right) c_{0}=\left(x_{n+1}-x_{n}-w_{n}\right) c_{l}+w_{n} c_{h} .
$$

Using now the spacing law (2) that has been established for this model one finds

$$
w_{n}=\frac{p\left(c_{0}-c_{l}\right)}{c_{h}-c_{l}} x_{n}=\zeta x_{n} .
$$

We have thus derived the width law and obtained the coefficient of proportionality, $\zeta$, as well. The importance of $\zeta$ lies in that measuring it provides a way of accessing $c_{0}$ that is not easily measured otherwise.

\section{FINAL REMARKS}

In summary, we have seen that the spinodal decomposition scenario for the formation of Liesegang patterns performs well whenever quantitative comparison with experiments is possible. It remains to be seen if the applicability of this model extends beyond the regular patterns. One should certainly try to use this theory to explain the exotic patterns (e.g. inverse patterns, helixes) that are experimentally reproducible and lack even qualitative understanding.

\section{ACKNOWLEDGMENTS}

I thank M. Droz, M. Zrínyi, T. Antal, P. Hantz, J. Magnin, and T. Unger for useful discussions. This work has been supported by the Hungarian Academy of Sciences (Grant No. OTKA T 029792).

[1] R. E. Liesegang, Naturwiss. Wochenschr. 11, 353 (1896).

[2] H. K. Henisch, Periodic precipitation (Pergamon Press, 1991).

[3] K. H. Stern, A Bibliography of Liesegang Rings, 2nd ed. U.S. Gov. Printing Office, Washington, (1967)

[4] G. T. Dee, Phys. Rev. Lett. 57, 275 (1986).

[5] B. Chopard, P. Luthi and M. Droz, Phys. Rev. Lett. 72, 1384 (1994); J. Stat. Phys. 76, (1994).

[6] T. Antal, M. Droz, J. Magnin, Z. Rácz and M. Zrínyi, J. Chem. Phys. 109, 9479, (1998).

[7] H. W. Morse and G. W. Pierce: Diffusion and Supersaturation in Gelatine, Proc. American Academy of Arts and Sciences, 38, 625-647 (1903)

[8] K. Jablczynski, Bull. Soc. Chim. France 33, 1592 (1923).

[9] R. Matalon and A. Packter, J.Colloid Sci. 10, 46 (1955).

[10] A. Packter, Kolloid Zeitschrift 142, 109 (1955).

[11] S. C. Müller, S. Kai, and J. Ross, J. Phys. Chem., 86, 4078 (1982). 
[12] M. Droz, J. Magnin and M. Zrínyi, J. Chem. Phys. 110 9618 (1999).

[13] C. Wagner, J. Colloid Sci., 5, 85 (1950).

[14] S. Prager, J. Chem. Phys. 25, 279 (1956).

[15] Ya. B. Zeldovich, G. I. Barrenblatt and R. L. Salganik, Sov. Phys. Dokl. 6, 869, (1962).

[16] T. Antal, M. Droz, J. Magnin, and Z. Rácz, Phys. Rev. Lett. 83, 2880 (1999).

[17] J.D. Gunton, M. San Miguel and P.S. Sahni, The $d y$ namics of first order transitions, in Phase transition and Critical Phenomena, Vol. 8, Ed. by C. Domb and J.L. Lebowitz, Academic Press (1983).

[18] L. Gálfi and Z. Rácz, Phys. Rev. A38, 3151 (1988).

[19] H. Larralde, M. Araujo, S. Havlin and H. E. Stanley, Phys. Rev. A46, 855 (1992).
[20] For usual parameter values, the width is much smaller than all the other length-scales and its actual value does not significantly affect the onset of phase separation.

[21] J. W. Cahn and J. E. Hilliard, J. Chem. Phys. 28, 258 (1958); J. W. Cahn, Acta Metall, 9, 795 (1961).

[22] P.C. Hohenberg and B.I. Halperin, Rev. Mod. Phys. 49, 435 (1977).

[23] S. Cornell and M. Droz, Phys. Rev. Lett. 70, 3824, (1993).

[24] The spinodal decomposition takes place in the regime where the homogeneous solutions of eq.(7) are linearly unstable.

[25] Y.-E. Lee Koo and R. Kopelman, J. Stat. Phys. 65, 893 (1991). 
This figure "fig1.jpg" is available in "jpg" format from: http://arxiv.org/ps/cond-mat/9910028v1 